\title{
Dossier
}

\section{Los desafíos de la perspectiva de género en la definición de la agenda gubernamental}

María Alejandra Ingaramo Lic. en Ciencia Política (UNR)

Postgrado en Planificación y Gestión de Políticas Sociales (UNR) Maestranda en Desarrollo Humano (FLACSO Argentina) Adscripta a la Cátedra de Política Social II (Esc. de Trabajo Social - UNR) E-mail: aleingaramo@yahoo.com 


\section{Introducción}

Nos interesa trabajar el proceso de formación de la agenda pública y el contexto donde el género se incorpora como problema y como perspectiva de intervención de las políticas públicas. La noción de política pública nos remite al Estado, a sus articulaciones con la sociedad, a las organizaciones públicas y a sus decisiones y actividades concretas. Las políticas públicas son comprensiones sociales relativamente compartidas, construidas a través de manifestaciones de propósitos y de su reformulación y reinterpretación dinámica, productos de un proceso que algunos autores denominan de retroalimentación y otros de reflexividad (Martínez Nogueira, 2012).

Incluir la perspectiva de género en el diseño e implementación de políticas públicas en América Latina implica reconocer que las culturas políticas históricamente dominantes se han creado sobre diferencias que estigmatizan al distinto (sea pobre, mujer, indio, mestizo, migrante, negro) limitando las posibilidades de desarrollo de las capacidades (sobre todo políticas) de las personas y de las comunidades. Existe en la región un patrón de exclusión social y negación del desarrollo de las capacidades humanas de larga duración que se expresa en prácticas y proyecciones de culturas políticas de la desigualdad (Calderón y Smuckler, 2002).

Los Estados latinoamericanos enfrentan el desafío de gobernar en contextos políticos plurales y autónomos, de alta intensidad ciudadana y con grandes problemas irresueltos (Aguilar Villanueva, 1996:30). El contexto planteado requiere cumplir dos requisitos fundamentales: gobernar por políticas y gobernar con sentido público administrando las tensiones que se producen al interior del tejido social.

La constitución de la ciudadanía en América Latina ha transitado y transita sobre la tensión entre un patrón de inclusión-exclusión: económica, política y social, dando como resultado una agenda pública desbordada de problemas que luchan por ingresar a la órbita estatal. 
La ciudadanía moderna está atravesada por la tensión entre la igualdad formal o jurídica y la desigualdad real o socioeconómica y es en esta tensión donde el género es un registro muy fuerte de desigualdad. Consideramos que en este contexto el Estado y sus políticas configurarán las relaciones de género al interior de la sociedad al mismo tiempo que las relaciones de género lo hacen con el tipo de Estado (Silveira, 2001:309).

Para lograr un desarrollo más equitativo y democrático se deben eliminar los tratos discriminatorios contra cualquier grupo. En el caso de las mujeres, el diseño de políticas que tomen en cuenta las condicionantes culturales, económicas y sociopolíticas que favorecen la discriminación femenina se ha convertido en un tema de agenda para los gobiernos (Lamas, 1996).

Nancy Fraser sostiene que para abordar la injusticia de género, se deben cambiar tanto la economía política como la cultura y desarmar la subordinación cultural y económica que organiza los espacios de reproducción de la sociedad. La desigualdad económica se encuentra en la división del trabajo productivo remunerado y el trabajo doméstico no remunerado y en la existencia de ocupaciones profesionales mejor pagas dominadas por los hombres y peor pagas ocupadas por las mujeres. El género no es sólo desigualdad política y económica sino también desigualdad cultural, por la existencia de modelos culturales dominantes de interpretación y valoración que privilegian rasgos asociados a la masculinidad (Fraser, 1996).

Incluir la perspectiva de género en los procesos de definición de las políticas públicas implica reconocer el enfoque de derechos como objetivo de las mismas, utilizando el marco conceptual que brindan los derechos humanos para aplicarlos a las políticas de desarrollo que deben considerar no sólo las obligaciones negativas del Estado sino también un conjunto de obligaciones positivas sobre aquello que debe hacer en orden a lograr la plena realización de los derechos civiles, políticos y también económicos, sociales y culturales (Pautassi, 2008).

En este sentido, Abramovich y Pautassi (2009) sostienen que en América Latina de forma paulatina va ganando consenso la nece- 
sidad de pensar a las políticas públicas en general y a las políticas sociales en particular como parte de las obligaciones estatales para el cumplimiento efectivo de los derechos humanos, permitiendo superar la visión de las políticas sociales bajo una lógica signada por la oferta de beneficios de tipo asistencial, para dirigirse a la definición de obligaciones estatales, mediante los distintos instrumentos que el Estado tiene a su alcance, superando la definición de los sujetos como meros beneficiarios.

Entendemos que las políticas públicas son inclusivas para la perspectiva de género cuando cuentan con la voluntad explícita de parte de las autoridades de promover una redistribución entre los géneros en términos de asignación de recursos, participación, derechos civiles, poder, autoridad y valoración del trabajo de hombres y mujeres (Silveira, 2001:309-310).

El estudio de los propósitos perseguidos por las políticas públicas constituye una instancia necesaria en la reconstrucción del rol del Estado como propulsor o freno a los cambios sociales. Por ello, las conceptualizaciones que se hagan de las cuestiones problemáticas no son inocentes, sino que conllevan supuestos, visiones que, desde su especificidad, contribuyen a remodelar (en lo concreto, material, pero también en cuanto a la estructuración de sentidos y visiones) esa sociedad en que se crean y desenvuelven (Meny y Thoenig, 2002).

\section{La construcción de la agenda de gobierno}

La definición del problema forma parte del proceso de decisiones de las políticas, donde determinados problemas son calificados como públicos y a otros se les niega tal calidad.

La agenda pública se compone de los hechos definidos como problemas por parte de la comunidad política y que son objeto de debate en la esfera pública, representando intereses y generando demandas de intervención estatal. En cambio, la agenda gubernamental está integrada por aquellas cuestiones que efectivamente han sido aceptadas 
por los decisores de política, convocan la atención estatal y generan políticas públicas (Cobb y Elder, 1986).

La cuestión, según Oszlak y O’ Donnell (1976), pasa porque ninguna sociedad posee la capacidad ni los recursos para atender omnímodamente a la lista de necesidades y demandas de sus integrantes. Sólo algunas son "problematizadas", en el sentido de que ciertas clases, fracciones de clase, organizaciones, grupos o incluso individuos estratégicamente situados creen que puede y debe hacerse "algo" a su respecto y están en condiciones de promover su incorporación a la agenda de problemas socialmente vigentes.

En este sentido, la formación de la agenda comprende el proceso a través del cual ciertos problemas o cuestiones ingresan como posibles asuntos de política pública. Se señalan dos hechos que indican la importancia del proceso: 1) la capacidad de atención del gobierno es necesariamente limitada, siempre hay más asuntos que atender que tiempo para considerarlos; 2) los problemas de políticas públicas no son datos a priori sino que son el resultado de definiciones (Cobs y Elder, 1986:77).

Cuando se define un problema, se ponen de manifiesto los aspectos que resultan insatisfactorios y cuál es la salida deseable o la perspectiva desde la cual nos interesa transformar esa realidad insatisfactoria. En este sentido, los problemas no existen en tanto tales sino que requieren de la percepción de un actor que plantee la cuestión, expresando los ámbitos de pertenencia de los actores donde estos se forman e informan (Díaz, 1998).

La construcción de la agenda pública es un proceso por el cual ciertos problemas o cuestiones reciben la atención activa del gobierno y donde la forma de ingreso de los temas puede determinar su tratamiento y su procesamiento futuro, la definición de su naturale$\mathrm{za}$, los actores participantes, las estrategias de implementación, etc. (Villanueva, 1996).

Definir una situación como un problema de política significa aceptar que tiene solución y delimitar sus soluciones posibles dentro de las competencias estatales. La calidad de problema de un fenómeno 
depende de la posibilidad de percibir su solución y ordenar su complejidad (Cobs y Elder, 1986:79).

Podemos citar como ejemplo dos cuestiones en relación a la problemática de la salud sexual y reproductiva de las mujeres donde encontramos que su tratamiento e ingreso a la agenda gubernamental está mediado por la influencia de decisores gubernamentales y no gubernamentales que inciden en la definición de la política produciendo respuestas limitadas en relación a las demandas de los actores sociales que han impulsado e impulsan su tratamiento en la esfera estatal.

La salud sexual y los derechos reproductivos son esenciales para permitir a las mujeres el goce de otros derechos y generar empoderamientos y participación en la vida económica y política de sus comunidades. La salud sexual y reproductiva y los derechos reproductivos son esenciales para el desarrollo sostenible. Todas las mujeres tienen que tener acceso a la planificación familiar voluntaria libre de coacción y discriminación ${ }^{1}$.

El movimiento feminista organiza sus demandas sobre la redefinición de la ciudadanía a partir de conceptos no sexistas e incorporando la categoría género en el debate del Estado propugnando el derecho a la diferencia. Entre los nuevos derechos reclamados se encuentran los derechos reproductivos, considerados como la base misma de la posibilidad de participación efectiva de las mujeres en la sociedad civil y en la vida política (Pautassi, 2000:14).

Una cuestión que forma parte de la agenda gubernamental en Argentina es la política nacional de salud sexual y procreación responsable ${ }^{2}$, que tiene como objetivos disminuir la morbimortalidad materno-infantil, prevenir embarazos no deseados, promover la salud sexual de los adolescentes, contribuir a la a prevención y detección precoz de enfermedades de transmisión sexual, garantizar el acceso

1 Declaración de Michelle Bachelet, Directora Ejecutiva de ONU Mujeres, en el Panel sobre Garantizar la equidad y la promoción de los derechos, inclusive para los jóvenes, Londres, 11 de julio de 2012. En www.unwomen.org/es/2012/07/ensuring-equity-and-promoting-rights

2 Ley nacional 25.673, 26 de mayo de 2003. Programa Nacional de Salud Sexual y Procreación Responsable. En www.msal.gov.ar/saludsexual/ley.php 
a la información, potenciar la participación femenina en la toma de decisiones relativas a su salud y procreación responsable.

El tratamiento y la formulación de la política nacional de salud sexual y procreación responsable exhibe una modalidad implícita de acción política que preserva y protege mediante garantías de salud pública solamente a la mujer madre. Si bien en el discurso esta acción pública pareciera ampliar las facultades ciudadanas de todas las mujeres en la esfera de la salud su racionalidad política e instrumental no garantiza beneficios a todas por igual (las que desean y no desean ser madres), sino que restringe derechos personalísimos de libertad excluyendo a las mujeres que no quieren serlo (Levín et al, 2007:205).

Esta situación explica en parte el conflicto de intereses que persiste sobre los derechos de las mujeres de decidir ser o no ser madres de acuerdo a su voluntad. La ausencia en la agenda gubernamental de políticas que permitan concretar la anticoncepción reversible o irreversible como prestación pública de salud y el acceso universal a la prestación médica que surja en consecuencia (Levín et al, 2007:231) es el producto de la tensiones y conflictos entre actores sociales y estales donde determinados grupos logran imponer sus cosmovisiones.

En la misma línea las demandas sobre la problemática de la despenalización/legalización del aborto $^{3}$ se instalan en la agenda pública desde hace más de tres décadas, es reconocida como un problema de salud pública, de justicia social y de ejercicio activo de la ciudadanía.

Los derechos sociales y la voluntad de conseguirlos son los principios fundamentales que orientan las conductas de los actores sociales. La defensa de la identidad, del control de su propia historia de vida, de su capacidad de negociar, de organizarse, de formular

3 En marzo de 2012 se presenta por cuarta vez en la Cámara de Diputados de la Nación el proyecto de ley de interrupción voluntaria del embarazo (IVE). La iniciativa consagra fundamentalmente: 1) el derecho de toda mujer a interrumpir voluntariamente un embarazo hasta las doce semanas del proceso gestacional; y 2) el derecho a acceder a la práctica del aborto en los servicios del sistema de salud pública de forma gratuita y en los servicios privados que las deben incorporar a sus prestaciones. Más de 250 organizaciones de mujeres, sociales, sindicales y de derechos humanos junto a la firma de 45 diputados/as consensuaron la iniciativa para que se dé el debate en comisiones y adquiera estado parlamentario nuevamente. En www.pagina12.com.ar/imprimir/diario/elpais/1-142177-2010-03-17. html y www.abortolegal.com.ar. Julio de 2012. 
proyectos, de impulsar cambios, revela la capacidad de los actores sociales de construir e incidir en la agenda pública (Touraine, 2000).

Las políticas de género son herederas de la lucha del feminismo y los movimientos sociales de mujeres por la igualdad, el reconocimiento y la libertad en función del ejercicio de sus derechos ciudadanos. El ejercicio de la militancia en la búsqueda de la igualdad habilita posibilidades que no dependen sólo del reconocimiento normativo o la posición legal de cada individuo, sino de la disponibilidad de una serie de recursos y capacidades intelectuales, sociales y culturales, conocimientos, recursos financieros, poder de movilización, negociación, etc. (Pautassi, 2008).

En torno a la distribución del poder político se observan tramas burocráticas y grupos de presión que funcionan sobre la estratificación social. En este sentido el análisis de la desigualdad es central para analizar situaciones de privación y de destitución (Sen, 2006:5).

En las sociedades industrializadas (y en otras), nadie duda de la importancia del movimiento de las mujeres que luchan no sólo por la igualdad de derechos y situaciones, sino sobre todo por su libertad. Las organizaciones de mujeres conforman un colectivo que actúa con otros actores, que cuentan con la capacidad de transformar su situación y su entorno (Touraine, 2005).

Que un tema ingrese a la agenda pública es el resultado del trabajo y la lucha de actores sociales funcionando en el marco del sistema político democrático, donde las decisiones no se alcanzan a través de la fuerza de la violencia, sino a través de la fuerza de la argumentación y el debate en el espacio público. La democracia es el gobierno de la discusión (Dréze y Sen, 2002:379).

En los sistemas democráticos el ejercicio de los derechos civiles y políticos brindan a la personas elementos para conocer las necesidades generales y demandar la intervención pública mediante acciones directas como votar, protestar y criticar, e incidir en la agenda de los gobiernos (Deneulin, 2009:10). Es en el marco de los sistemas democráticos donde las personas ejercen su agencia a través de la participación política y la toma democrática de decisiones, donde el razonamiento público constituye la esencia de la democracia. 
Las políticas estatales se definen como los "acordes" de un proceso social tejido alrededor de un tema o cuestión, que adquieren significación sólo y en la medida en que son sistemáticamente vinculadas al tema o cuestión que las origina, al ámbito de acción social en que se insertan, a los actores que intervienen en el proceso de "resolución" de la cuestión y a sus respectivas y sucesivas políticas. Observar las articulaciones que se establecen nos posibilita observar el Estado, las clases y grupos o coaliciones políticas "en movimiento". La implementación de las políticas estatales nos ofrece una visión del Estado “en acción” (Oszlak y O’Donnell, 1976).

Toda política pública encubre una teoría del cambio social porque está contenida en una relación causa y efecto entre las disposiciones que rigen y fundamentan la acción gubernamental. El decisor gubernamental se comporta como un operador que apuesta que cuando intervenga se producirá una consecuencia sobre el tejido social real sobre el que se desarrollará la acción (Meny y Thoenig, 1992:96-97).

Los programas y proyectos puestos en marcha constituyen cristalizaciones institucionales de las interpretaciones que los actores públicos realizan de la o las cuestiones problemáticas, es decir, son las interpretaciones acerca del "problema" y sus posibles vías de resolución o neutralización por parte del Estado, que orientan las modalidades de intervención adoptadas (Soldano y Andrenacci, 2006).

\section{Políticas públicas con género}

Por política pública entendemos un campo de acción del Estado, una declaratoria de intención, un proceso de conversión y ciclo de acción y un conjunto de impactos (Martínez Nogueira, 2006). Pautassi (2008) plantea que si bien existen contextos más propicios que otros para la implementación de políticas públicas respetuosas de los derechos humanos donde la variabilidad está dada por el papel de los actores en el proceso, es necesario distinguir los pasos necesarios para la elaboración de políticas públicas transversales y con enfoque de derechos que promuevan la equidad social y de género. 
Ana Laura Rodríguez Gustá (2008) aborda las diversas respuestas donde el género se convierte en cuestión de política pública. Analiza las características de cuatro políticas sensibles al género: a) las políticas de acción afirmativa, b) las políticas para las mujeres, c) las políticas con perspectiva de género y d) las políticas de transversalización de género. Nos interesa señalar en qué consiste cada una, para luego abordar específicamente las políticas de transversalización y la relevancia de la construcción de consensos en el proceso de definición del problema para la creación de condiciones de viabilidad en función de la multiplicidad de actores que intervienen en la implementación.

Las políticas de acción afirmativa promueven la presencia femenina en los espacios de decisión estatal, rescatando el hecho de las desventajas femeninas y la obligación del estado de garantizar mediante disposiciones específicas (cuotas/cupos) el ejercicio de una ciudadanía plena. Las políticas para las mujeres mantienen las diferencias primarias de roles entre varones y mujeres y sus objetivos se traducen en acciones compensatorias reconociendo la doble inserción femenina en el ámbito público y privado. Por otra parte, las políticas con perspectiva de género trabajan en la profundización de acciones para transformar las relaciones jerárquicas de género, implementando acciones concernientes a los derechos de las mujeres, la democratización de las familias y la corresponsabilidad en la reproducción y el cuidado al interior de la esfera doméstica (los planes de igualdad son un ejemplo de este enfoque).

Por último, las políticas de transversalización de género, a diferencia de las políticas reseñadas en el párrafo anterior, implican la integración sistemática de la igualdad en todos los sistemas y estructuras, políticas y procedimientos, representando un desafío para la definición de la agenda pública porque demanda articulaciones políticas e institucionales virtuosas donde el Estado debe contar con capacidades burocráticas afinadas, implicando además que asumir la perspectiva de la transversalización en las políticas públicas significa que las construcciones culturales en torno a lo femenino y lo masculino están cristalizadas también en las estructuras y prácticas del propio Estado (Rodríguez Gusta, 2008:115). 
Un enfoque de institucionalización y de transversalidad, señala Pautassi (2008), no debe situarse como una integración o como una sumatoria de estrategias y estructuras preexistentes, sino que se requieren cambios fundamentales coordinados en: la política, la cultu$\mathrm{ra}$, las estrategias y las prácticas organizacionales.

Las políticas de transversalización consisten en la promoción de la igualdad de género mediante su integración sistemática en todos los niveles, estructuras, procesos, procedimientos y culturas organizacionales. Implican intervenciones diseñadas sobre un conjunto de funciones, fines y consecuencias, que ponen en cuestión la necesidad de instrumentar acciones específicas que permitan la inclusión de la perspectiva de género en todos los espacios de gestión del gobierno involucrando al conjunto de actores estatales, políticos y sociales.

Fraser reconoce que el género es una colectividad bivalente que abarca dimensiones económicas y culturales y que para la comprensión del carácter de la injustica de género se necesita prestar atención a la redistribución y al reconocimiento. Un concepto bivalente de justicia abarca a la vez la redistribución y el reconocimiento sin tener que reducirse uno en otro (Fraser, 1996:23). La autora trabaja la noción de paridad participativa sobre normas básicas de igualdad legal formal y dos precondiciones adicionales, la primera, la distribución de recursos materiales para asegurar la independencia y la voz, y la segunda, que los modelos culturales de interpretación y valoración permitan expresar el respeto mutuo para todos los participantes (Fraser, 1996:32).

Teniendo en cuenta las relaciones de poder a las que los individuos y actores están sometidos, sería posible lograr una relación entre igualdad y diferencia en la medida en que se refuerce una cultura deliberativa que no sólo permita la canalización institucional de la participación ciudadana sino que estimule la participación de individuos y actores. La agenda y la solución de problemas se constituyen a partir de la deliberación pública de los participantes, colectivamente a través de la argumentación y contra-argumentación entre los involucrados (Calderón y Smukler, 2004:24). 


\section{Los problemas de la implementación}

El éxito de las intervenciones dirigidas a remediar o superar situaciones de injustica como la pobreza, la exclusión, la vulnerabilidad o las discriminaciones o dependencias de todo tipo depende en gran medida de la implementación. Fijar la atención en la implementación implica adoptar una perspectiva teórica que privilegia el análisis de los procesos de producción de impactos sobre la sociedad de la acción política (Martínez Nogueira, 2004:2).

El proceso de implementación de políticas transversales es complejo, implica muchos actores, decisiones encadenadas y alto nivel de organización y conocimiento. Aguilar Villanueva (1996) señala que es necesario otorgar autonomía a la burocracia para generar las condiciones necesarias para generar políticas con calidad.

La implementación necesita ser diseñada y programada teniendo en cuenta la existencia de lógicas institucionales y la complejidad de la cooperación. Frente a la eficacia y la eficiencia encontramos el déficit y las imperfecciones. La cantidad de puntos de decisiones y de actores es un requisito fundamental que las burocracias cuenten con autonomía para la implementación adaptativa por casos y contextos.

La inercia organizacional en la gestión de las políticas públicas revela la necesidad de fortalecer la burocracia para actuar transversalmente. El Estado necesita capacidades burocráticas afinadas, prácticas formalizadas de planificación, presencia de personal estable entrenado, instancias de coordinación y articulación, producción de información y comunicación donde la interlocución con las organizaciones de mujeres presupone modalidades ampliadas de articulación entre el Estado y la sociedad civil (Rodríguez Gustá, 2008:125).

Las políticas transversales se integran con el análisis y el diseño de políticas públicas y son definidas por estrategias de acción entre gobierno y sociedad y un estilo de gestión diferenciado que incorpora la opinión, participación y corresponsabilidad de todos los actores implicados. Es decir políticas compatibles con el marco constitucional sustanciadas con la participación intelectual, la coordinación administrativa y la práctica ciudadana (Aguilar Villanueva, 1996:33). 
En este sentido, las políticas transversales necesitan generar acuerdos. El problema central de la implementación no reside en asegurar que quienes implementan se ajusten al plan de acción establecido sino en que el proceso de implementación permita alcanzar un mínimo consenso en torno a las metas así como un nivel de autonomía individual y un grado de compromiso con el plan de acción por parte de quienes deben implementar la política (Martínez Nogueira, 2004).

La inclusión supone reconocer las diferencias en los planos culturales, sociales y económicos, pero también construir un plano de igualdad política y jurídica en el que las distintas libertades se puedan desarrollar en el espacio público (Calderón, 2008:54).

\section{Consideraciones finales}

A lo largo de este trabajo hemos discutido el proceso de formación de la agenda pública y el contexto donde el género se incorpora como problema y como perspectiva de intervención de las políticas públicas.

En relación a la formación de la agenda encontramos que el ingreso de los problemas a la agenda gubernamental está mediado por las definiciones que actores gubernamentales y no gubernamentales imponen sobre las cuestiones y que determinan su formulación e implementación futura. Los actores sociales manifiestan sus necesidades y organizan la participación sintetizando las demandas de políticas públicas y luchando con otros por ocupar el espacio público.

La construcción de la agenda es un campo de lucha por imponer soluciones a cuestiones que surgen del ejercicio de la ciudadanía en sistemas democráticos complejos donde el Estado es la instancia donde se organizan e institucionalizan un número finito de cuestiones.

En este escenario el género funciona como un registro muy fuerte de desigualdad donde se cristalizan situaciones que deben ser removidas para garantizar los derechos de las mujeres y el ejercicio pleno de su libertad. Y, a su vez, organiza a un numeroso conjunto de mo- 
vimientos sociales que participan activamente por la promoción de esos derechos vulnerados.

Si bien hay registros de compromiso gubernamental en el desarrollo y fortalecimiento de políticas de género para la promoción de una democracia más igualitaria, las experiencias necesitan ser revisadas, si lo que buscan es lograr efectivamente la consolidación de los derechos humanos en general y de las mujeres en particular.

Asimismo, formular e implementar políticas sensibles al género como herramientas de intervención al interior del Estado implica romper con funcionamientos inerciales al interior del propio Estado. Dentro de las esferas estatales los funcionamientos están condicionados por las capacidades institucionales, que repercuten en la calidad de las políticas, donde respuestas heterogéneas y aisladas no alcanzan para establecer transformaciones que rompan con las tramas de la desigualdad.

Como decíamos en la introducción: el Estado y sus políticas contribuyen a configurar las relaciones de género al interior de la sociedad al mismo tiempo que las relaciones de género lo hacen con el tipo de Estado (Silveira, 2001). El enfoque de género se emparenta con el enfoque de derechos cuando las diferencias de género son concebidas como una injusticia que implica la intervención estatal para resolverla (Pautassi, 2008). La cuestión sigue pasando por la forma y, en consecuencia, por la oportunidad de las soluciones. 


\section{Referencias bibliográficas}

ABRAMOVICH, V. y PAUTASSI, L. "El enfoque de derechos y la institucionalidad de las políticas sociales", en: PAUTASSI, L. y ABRAMOVICH, V. (Compiladores). $L a$ revisión judicial de las políticas sociales. Buenos Aires, Editores del Puerto, 2009. pp. $279 / 340$.

AGUILAR VILLANUEVA, L. F. "Estudio introductorio”, en: Antología de políticas públicas, Vol. 1: El estudio de las políticas públicas. México, Miguel Ángel Porrúa Grupo Editorial, 1992.

CALDERÓN, F. “Ciudadanía y Desarrollo Humano”, en: CALDERÓN, F. (coordinador). Ciudadanía y Desarrollo Humano. Cuaderno de Gobernabilidad Democrática N 1 . Nueva York-Buenos Aires, PNUD-Siglo XXI, 2007.

CALDERON, F y SZMUKLER, A. "Political Culture and Development", en: RAO y WALTON (Eds.). Culture and Public Action. Stanford, California, Stanford University Press, 2004. pp. 281-306. Versión en español.

DENEULIN, S. y SPENCE, R. “Desarrollo Humano y Políticas Públicas”, en: Una introducción al enfoque del desarrollo humano y las capacidades. Publicado en inglés por Earthscan en el Reino Unido y Estados Unidos, 2009. Traducción: Adriana Velasco, Programa de Posgrado de FLACSO Argentina, 2009.

DENEULIN, S. “Democracia y Participación Política”, en: Una introducción al enfoque del desarrollo humano y las capacidades. Publicado en inglés por Earthscan en el Reino Unido y Estados Unidos, 2009. Traducción: Adriana Velasco, Programa de Posgrado de FLACSO Argentina, 2009.

DÍAZ, C. "El ciclo de las políticas públicas locales: notas para su abordaje y reconstrucción”, en: VENESIA, J. (comp.). Políticas públicas y desarrollo local. Rosario, Instituto de Desarrollo Regional, 1998.

DREZE, J. y SEN, A. Democratic Practice and Inequality in India: Development and Participation. Delhi, Oxford University Press, 2002.

ELDER, C. y COBB, R. "Formación de la agenda. El caso de la política de los ancianos”, en: Aguilar Villanueva, L. Antología de políticas públicas, Vol. 3: Problemas públicos y agenda de gobierno. México DF, Miguel Ángel Porrúa, 1993.

FRASER, N. "Redistribución y Reconocimiento: hacia una visión integrada de justicia del Género", en: Revista Internacional de Filosofía Politica $\mathrm{N}^{\circ}$ 8. Universidad Nacional de educación a distancia Universidad Autónoma Metropolitana. Madrid, 1996. pp. $18-40$.

LAMAS, M. “La perspectiva de género”, en: Revista La Tarea. № 8, México, 1996. Disponible en: http://www.latarea.com.mx/articu/articu8/lamas8.htmhttp. 
LEVÍN, S y otras. "La política de género de las políticas públicas en Argentina”, en: Carbonero M.A. y Levín S. (comp.) Entre Familia y Trabajo. Relaciones, conflictos y políticas de género en Europa y América Latina. Rosario, Homo Sapiens Ediciones, 2007.

MARTÍNEZ NOGUEIRA, R. "Desafíos estratégicos en la implementación de políticas sociales". Presentado en el Encuentro Hacia una visión estratégica de la implementación de programas sociales, organizado por el Banco Interamericano de Desarrollo, Washington, noviembre 2004.

MARTÍNEZ NOGUEIRA, R. "Heterogeneidad y contingencia en los programas sociales: consecuencias para la gestión de la implementación”, en: Reforma $y$ Democracia. $\mathrm{N}^{\circ}$ 35. Caracas, CLAD, 2006.

MENY, Y. y THOENG, J. Las Políticas Públicas. Barcelona, Ariel Ciencia Política, 1992.

OSZLAK, O y O'DONNELL, G. "Estado y políticas estatales en América Latina: hacia una estrategia de investigación”, en: Acuña, C. (comp.) Lecturas sobre el Estado y las políticas públicas: retomando el debate de ayer para fortalecer el actual. Proyecto de Modernización del Estado, Jefatura Gabinete de Ministros, Buenos Aires, 2008.

PAUTASSI, L. "Igualdad de Derechos y Desigualdad de Oportunidades. Ciudadanía, Derechos Sociales y Género en América Latina”, en: Herrera, G (comp). Las fisuras del patriarcado. Reflexiones sobre feminismo y derecho. Quito, FLACSO-CONAMU, 2000.

PAUTASSI, L. Enfoque de derechos y transversalidad de género en la política pública: un camino por recorrer. Publicación digital, CD Rom. IX Jornadas de Historia de las Mujeres y IV Congreso Iberoamericano de Estudios de Género. Rosario, 2008.

RODRIGUEZ GUSTÁ, A. "Las políticas sensibles al género: variedades conceptuales y desafíos de intervención", en: Revista Temas y Debates. Año 12, $\mathrm{N}^{\circ} 16$. Facultad de Ciencia Política y RR.II, Rosario, UNR Editora, 2008. pp. 109/129.

SEN, A. "Foreword to Pathologies of Power", preface to Farmer, P. Pathologies of Power. University of California Press, 2006.

TOURAINE, A. "Los derechos culturales", en: Un nuevo paradigma para el mundo de hoy. Buenos Aires, Paidós, 2005. pp. 181-227.

TOURAINE, A. "El sistema y los actores", en: Revista del CLAD Reforma y Democracia. $\mathrm{N}^{\circ} 18$, Caracas, 2000. pp. 1-9. 


\section{a. Libros:}

APELLIDO, Nombre (del/a autor/a del libro). Título del libro. Lugar, Editorial, año.

b. Artículos o Capítulos de Libros:

APELLIDO, Nombre (del/a autor/a del artículo o capítulo). “Título del artículo o capítulo", en: APELLIDO, Nombre (del/a autor/a, compilador/a, organizador/a o editor/a del libro). Título del libro o revista. Lugar, Editorial, año. Páginas (del artículo o capítulo).

\section{c. Artículos de Publicación Periódica:}

APELLIDO, Nombre (del/a autor/a del artículo o capítulo). “Título del artículo o capítulo", en: Título de la Publicación. Año, Volumen, Número, $\mathrm{N}^{\circ}$ 37. Páginas (del artículo).

\section{d. Fuentes y/o documentos electrónicos:}

APELLIDO, Nombre (del/a autor/a del libro, artículo, ponencia, etc.). Título. Tipo de soporte (CD-ROM; página web; blog). Fecha de publicación. ISBN o ISSN [en línea: fecha de consulta]. Disponible en: link completo.

\section{Sistema de Arbitraje}

Para la aprobación de su publicación, los trabajos serán sometidos a la consideración del Consejo de Redacción y de evaluadores/as externos/as. Se tendrá en cuenta para su aprobación la pertinencia del tema propuesto, la calidad de la publicación, la actualización de la bibliografía y el cumplimiento de normas formales de redacción. En primer lugar, los artículos serán objeto de una evaluación preliminar por parte del Consejo de Redacción, quien determinará la pertinencia para su publicación. Una vez establecido que el artículo cumple tanto con los requisitos temáticos como formales, será enviado a dos pares académicos externos, quienes determinarán, de manera anónima: a) recomendar la publicación sin modificaciones; b) publicar con sugerencias de re-elaboración; c) no recomendar la publicación. Los resultados del proceso de evaluación serán inapelables en todos los 
casos. En caso de ser publicado el artículo, el/los autor/es transfieren todos los derechos de autor a Cátedra Paralela, sin cuyo permiso expreso no podrá reproducirse ninguno de los materiales allí publicados. Asimismo, Cátedra Paralela asume los derechos para editar, publicar, reproducir, distribuir copias, preparar trabajos derivados en papel y/o electrónicos e incluir el artículo en índices nacionales e internacionales o bases de datos. Se solicita a los/as colaboradores/as la entrega de la nota expresa de originalidad y cesión de los derechos de autor/a. La Revista Cátedra Paralela no se hace responsable por los trabajos no publicados ni se obliga a mantener correspondencia con los/as autores/as.

\section{Modelo Nota de Autorización - Revista Cátedra Paralela}

Por la presente autorizo a la Revista Cátedra Paralela, a la publicación del artículo de mi autoría

.........en el $\mathrm{N}^{\circ}$....... del año ......, publicación de la Escuela de Trabajo Social de la UNR y el Colegio de profesionales de Trabajo Social de la $2^{a}$ Circunscripción de la Provincia de Santa Fe.

Se deja constancia de que no corresponde retribución pecuniaria derivada del derecho de autor.

LUGAR Y FECHA:

FIRMA Y ACLARACIÓN: 